Luiz Antonio dos Anjos ${ }^{\mathrm{a}}$

(iD) https://orcid.org/0000-0002-5257-6912

Thais Vieira Esteves ${ }^{\mathrm{b}}$

(iD) https://orcid.org/0000-0002-1230-6328

Virginia Gaissionok Mariz ${ }^{\mathrm{a}}$

(iD) https://orcid.org/0000-0001-9442-4530

Mauricio Teixeira Leite de

Vasconcellos ${ }^{\mathrm{c}}$

(iD) https://orcid.org/0000-0003-1658-2589

a Universidade Federal Fluminense, Laboratório de Avaliação Nutricional e Funcional, Departamento de Nutrição Social. Niterói, RJ, Brasil.

b Fundação Oswaldo Cruz, Centro de Estudos da Saúde do Trabalhador e Ecologia Humana, Escola Nacional de Saúde Pública Sergio Arouca. Rio de Janeiro, RJ, Brasil.

' Instituto Brasileiro de Geografia e Estatística, Escola Nacional de Ciências Estatísticas. Rio de Janeiro, RJ, Brasil.

Contato:

Luiz Antonio dos Anjos

E-mail:

lanjos@ig.com.br

Os autores declaram que o trabalho não foi subvencionado e que não há conflitos de interesses.

Luiz Antonio dos Anjos recebeu bolsa de produtividade em pesquisa pelo CNPq (Proc. 310461-2016-2). Virginia Gaissionok Mariz recebeu bolsa PIBIC/ CNPq (Proc. 127593/2014-4”.

Os autores informam que o trabalho não é baseado em tese ou dissertação e que foi apresentado no VII Congreso Latinoamericano de Nutrición, em Punta Cana, República Dominicana, 8-12 novembro de 2015.

Recebido 06/05/2016

Revisado: 14/03/2017

Aprovado: 11/05/2017

\section{Tendência do nível de atividade física ocupacional e estado nutricional de adultos ao longo de quatro décadas no Brasil}

\author{
Trend of adults' occupational physical activity levels and \\ nutritional status over four decades in Brazil
}

\section{Resumo}

Objetivo: avaliar a tendência do nível de atividade física ocupacional (Nafo) e o estado nutricional (EN) de adultos ( $\geq 20$ anos) em pesquisas nacionais brasileiras realizadas ao longo de quatro décadas. Métodos: os dados declarados da ocupação principal foram estratificados em três Nafo (leve, moderado e pesado) segundo as classificações vigentes à época das pesquisas. $\mathrm{O} E N$ foi determinado segundo a classificação da OMS baseada no índice de massa corporal (IMC, kg. ${ }^{-2}$ ) como baixo peso (IMC $<18,5)$, adequado $(18,5 \leq$ IMC $<25$ ) e sobrepeso (IMC $\geq 25$ ). Todas as estimativas foram calculadas usando os pesos amostrais das pesquisas. Resultados: a população com ocupação declarada aumentou no período, com mais Nafo moderado nos anos 1970 e migrando para Nafo leve ao final dos anos 2000. A prevalência de baixo peso diminuiu e a de sobrepeso aumentou substancialmente para todas as categorias de Nafo. O sobrepeso variou de 27,6 a 49,7\% no Nafo leve e de 12,7 a $43,3 \%$ no Nafo pesado. Conclusões: apesar da limitação do estudo (atividade física estimada pela ocupação declarada sem considerar a evolução/modificação dos processos de trabalho), conclui-se que os trabalhadores no Brasil apresentam balanço energético positivo independente do Nafo.

Palavras-chave: saúde do trabalhador; sobrepeso; índice de massa corporal; inquéritos epidemiológicos; atividade motora.

\begin{abstract}
Objective: to evaluate the trend of adults' ( $\geq 20$ years) occupational physical activity levels and nutritional status in Brazilian national surveys carried out over four decades. Methods: the declared data regarding main occupation was stratified in three physical activity levels (light, moderate, and heavy) according to the classifications in force when the surveys were conducted. The nutritional status was determined according to the World Health Organization classification based on the body mass index (BMI, $\left.\mathrm{kg} \cdot \mathrm{m}^{-2}\right)$ as underweight (BMI < 18.5), normal $(18,5 \leq I M C<25)$ and overweight $(B M I \geq 25)$. All estimates were calculated using the sampling weights from the surveys. Results: the population with declared occupation increased in the period, with greater moderate physical activity levels in the 1970's and shifting to light in the 2000's. The prevalence of underweight decreased and of overweight increased substantially for all physical activity levels. Overweight ranged from 27.6 to $49.7 \%$ in light levels and from 12.7 to $43.3 \%$ in heavy levels. Conclusions: despite the limitations of the study (the physical activity levels were estimated by declared occupation and did not consider the development/modification of labor processes), we concluded that workers in Brazil present a positive energy balance regardless of their occupational physical activity levels.
\end{abstract}

Keywords: occupational health; overweight; body mass index; epidemiological surveys; motor activity. 


\section{Introdução}

O perfil nutricional da população mundial vem se modificando no decorrer dos últimos anos em função do aumento dos índices de sobrepeso e obesidade, particularmente em países em desenvolvimento $^{1,2}$. De fato, sobrepeso pode ser considerado atualmente como o mais importante agravo nutricional na população mundial ${ }^{3}$. Segundo a Organização Mundial da Saúde (OMS), cerca de 2,5 bilhões de adultos no mundo tinham sobrepeso/obesidade em $2018^{3}$. Associada a outras tendências (envelhecimento, urbanização) a mudança no perfil nutricional faz com que uma nova agenda de prioridades de ação na área da Saúde seja pensada ${ }^{4}$. Dados brasileiros indicam quadro semelhante ao do resto do mundo de aumento do sobrepeso e da obesidade e diminuição da desnutrição em sua população nas últimas décadas ${ }^{5,6}$. Na mais recente Pesquisa de Orçamentos Familiares 2008-2009, a prevalência de sobrepeso/ obesidade foi de $49 \%$ nos brasileiros com 20 anos ou mais de idade ${ }^{6}$.

Do ponto de vista etiológico, em nível populacional, o sobrepeso é causado por quadro de balanço energético (BE) positivo decorrente de aumento na ingestão energética (IE) ou de redução do gasto energético (GE), por redução da atividade física em um ou mais de seus domínios (doméstico, ocupacional, lazer ou transporte) ou uma combinação desses componentes ${ }^{7}$. O GE de um indivíduo é composto pela (1) taxa metabólica basal (TMB), que é o mínimo de energia que o organismo necessita para seu funcionamento em condição basal; (2) energia necessária para a digestão, absorção e utilização dos nutrientes e (3) atividade física, que pode ser definida como qualquer movimento realizado pela musculatura esquelética que resulte em $\mathrm{GE}^{8}$, compreendendo, assim, os domínios de afazeres domésticos, ocupacionais, de lazer e de transporte ${ }^{4}$.

O perfil nutricional da população brasileira atual pode ser justificado pelo aumento das novas tecnologias após a Segunda Guerra Mundial, uma vez que a industrialização visava à produção em massa de produtos padronizados de baixo custo como eletrodomésticos e automóveis, além da expansão do setor da alimentação através das descobertas da tecnologia de alimentos com produtos semiprontos e de fácil preparo, diminuindo, assim, a atividade física praticada com afazeres domésticos ${ }^{4,9}$. O consumo elevado de produtos industrializados, particularmente os ultraprocessados que alteram vários componentes dos alimentos ${ }^{10}$, é apontado como o maior causador do aumento energético na dieta ${ }^{11}$. Além disso, o perfil ocupacional também foi alterado em razão da migração da população de área rural para a área urbana, ocasionando tendência de diminuição no número de ocupações pesadas, como a indústria e a agricultura, e aumento de ocupações com nível de atividade física (NAF) leve, como a área de serviços ${ }^{4}$. Outra questão relevante é a inserção crescente de mulheres no mercado do trabalho, sendo observadas mudanças no padrão alimentar familiar com o aumento na ingestão de alimentos de baixo valor nutritivo e fora do domicílio, e diminuição do GE em atividade física não-ocupacional (doméstica, lazer, transporte) ${ }^{4}$, contribuindo para o aumento da obesidade na população feminina.

No Brasil, não existe estudo de abrangência nacional e base domiciliar que tenha investigado o NAF da população ${ }^{12}$. Em 1997, pesquisa realizada em amostra probabilística da população do Nordeste e do Sudeste do Brasil indicou que $20 \%$ da população praticavam exercício físico ou esporte ${ }^{4}$, com grande diferença entre homens $(27,3 \%)$ e mulheres $(13,1 \%)$. Ao se incorporar as informações sobre a atividade física ocupacional (AFO), o número de indivíduos ativos aumentou substancialmente. Entre o total que não praticava exercício físico ou esporte, 23,0 e 49,3\% tinham nível de atividade física ocupacional (Nafo) pesado e moderado, respectivamente. Assim, o total de ativos sobe para $44,2 \%$, se acrescentados os indivíduos com Nafo moderado ${ }^{12}$. Apesar da limitação na pergunta sobre a prática de atividade física, esta foi a primeira pesquisa de base populacional em regiões brasileiras que obteve tal informação ${ }^{12}$.

Esses dados indicam que o Nafo ainda é um importante componente da atividade física total da população brasileira. Dessa forma, a investigação do Nafo e do estado nutricional (EN) da população brasileira adulta ocupada parece apropriada na perspectiva de melhor entendimento da relação entre o estado nutricional e as características ocupacionais da população. Neste contexto, este estudo investigou as tendências do Nafo e do EN da população brasileira ao longo de quatro décadas, a partir dos anos 1970, através de análises de quatro pesquisas populacionais de abrangência nacional que coletaram medidas antropométricas e informações de ocupação.

\section{Métodos}

A análise foi baseada nos dados do Estudo Nacional da Despesa Familiar (Endef) realizado na década de 1970, da Pesquisa Nacional de Saúde e Nutrição (PNSN) realizada em 1989 e das duas mais recentes Pesquisas de Orçamentos Familiares (POFs), realizadas em 2002-2003 e em 2008-2009. Todas as pesquisas foram realizadas pelo Instituto Brasileiro de Geografia e Estatística (IBGE) e os microdados das 
POFs estão disponíveis no portal do IBGE. Os microdados e documentação do Endef e da PNSN foram disponibilizados pelo IBGE em meio magnético.

O Endef foi uma pesquisa domiciliar sobre consumo de alimentos e orçamentos familiares, com coleta de dados entre agosto de 1974 e agosto de 1975. Uma amostra probabilística de cerca de $55 \mathrm{mil}$ domicílios foi selecionada em quatro estágios (municípios, setores censitários, subsetores e domicílios) ou três estágios (nos municípios mais populosos, ditos autorrepresentativos), para produzir estimativas para 22 estratos geográficos do país. A amostra fornece dados sobre 267.446 pessoas de 53.311 famílias $^{13}$.

A PNSN foi uma pesquisa encomendada pelo extinto Instituto Nacional de Alimentação de Nutrição e pelo Instituto de Planejamento Econômico e Social, com coleta de dados entre julho e setembro de $1989^{14}$. Uma amostra probabilística de 17.920 domicílios foi selecionada em três estágios (município, setor e domicílio) para representar nove estratos geográficos do país. Foram entrevistadas 63.213 pessoas moradoras.

As POFs foram realizadas em 2002-2003 e 20082009. Houve ainda POFs em 1987-1988 e 1995-1996, mas que não coletaram dados antropométricos e não tiveram abrangência nacional. Na POF de 20022003, chamada a partir de agora como POF-2002, a coleta de dados foi realizada entre julho de 2002 e junho de 2003, em uma amostra conglomerada em dois estágios (setores e domicílios), com estratificação geográfica e estratos socioeconômicos (média no setor dos anos de estudo dos responsáveis pelos domicílios) em 48.470 domicílios de 3.984 setores censitários $^{15}$. Já a POF de 2008-2009 (POF-2008), foi realizada entre 19 de maio de 2008 e 18 de maio de 2009, com procedimentos amostrais semelhantes à POF-2002, mas em 55.970 domicílios de 4.696 setores censitários ${ }^{16}$.

Para realizar as análises, foram utilizadas as informações de adultos ocupados com idade $\geq 20$ anos, excluindo-se as mulheres gestantes ou lactantes de todas as pesquisas, totalizando 72.988 , 20.672, 68.957 e 92.121 pessoas nas quatro pesquisas, por ordem cronológica. Foram selecionadas as variáveis de interesse que envolvem a ocupação e avaliação antropométrica: sexo, idade, massa corporal, estatura, código de ocupação, código de atividade econômica, macrorregião de origem e situação urbana ou rural. Tanto no Endef quanto na PNSN não se investigou a área rural da região Norte. No Endef, também não se investigou a área rural da região Centro-Oeste.

O estado nutricional foi estabelecido a partir do índice de massa corporal (IMC) definido como a divisão da massa corporal $(\mathrm{kg})$ pelo quadrado da estatura (m), usando os pontos de corte e a nomenclatura sugeridos pela $\mathrm{OMS}^{17}$ : baixo peso, IMC < 18,5; adequado, 18,5 $\leq$ IMC $<25$; e sobrepeso, IMC $\geq 25 \mathrm{~kg} \cdot \mathrm{m}^{-2}$. Optou-se por incluir obesidade (geralmente definida como IMC $\geq 30 \mathrm{~kg} \cdot \mathrm{m}^{-2}$ ) na categoria sobrepeso para fins práticos e de comparação com os vários estudos.

As pesquisas levantaram dados sobre as ocupações econômicas exercidas pelas pessoas, com pequenas diferenças nas instruções de coleta. Enquanto o Endef levantou todas as ocupações exercidas por todos os moradores durante os 12 meses anteriores à pesquisa ${ }^{15,16,18}$, na PNSN coletou-se informação sobre a ocupação exercida na semana anterior à coleta de dados. Nas POFs foram pesquisadas as ocupações que o entrevistado exerceu nos 12 meses anteriores à coleta no domicílio e a ocupação atual foi determinada como a principal, em caso de mais de uma ocupação simultânea considerou-se a que tinha maior número de horas no mês. Como, porém, foi determinada no Endef e nas POFs a ocupação que estava sendo exercida na semana anterior à pesquisa, denominada ocupação atual, as diferenças de coleta parecem não ser relevantes nesta análise.

As ocupações declaradas em cada pesquisa foram codificadas de acordo com a classificação das ocupações do IBGE, vigente à época de cada pesquisa, ou seja, a estrutura de códigos do Censo Demográfico de 1970, para o Endef, e as dos Censos Demográficos anteriores à realização de cada pesquisa. Nessas classificações, a cada código associa-se um rótulo (usado nas pesquisas) que objetiva dar uma descrição sumária do conjunto de ocupações do grupo. Assim, a associação entre os códigos das pesquisas, bem como a atribuição de um Nafo a cada código, passa pela comparação do conjunto de ocupações incluídas em cada código, para se estabelecer o Nafo que se refere à classificação das ocupações em grandes grupos que representam o GE associado ao seu exercício.

No Endef, todas as pessoas ocupadas da amostra tiveram suas ocupações classificadas segundo a atividade física a partir de classificações encontradas na literatura ${ }^{19}$. Na PNSN, a classificação de Nafo foi feita de acordo com os critérios, estabelecidos pela Organização das Nações Unidas para a Alimentação e a Agricultura (FAO) e pela OMS à época ${ }^{20}$, para os quais as ocupações leves são aquelas em que o trabalhador, durante seu trabalho, passa $75 \%$ do tempo sentado ou em pé e $25 \%$ em pé realizando atividades consideradas leves; no nível moderado o trabalhador passa $25 \%$ do tempo sentado ou em pé e $75 \%$ do tempo em atividade moderada; e nas ocupações pesadas o trabalhador passa $40 \%$ do tempo sentado ou em pé e $60 \%$ do tempo em atividade ocupacional específica. A intensidade das atividades 
ocupacionais específicas (leve, moderada ou pesada) é definida de acordo com estimativas do custo energético das atividades. A classificação de Nafo combinou os códigos de ocupação e a atividade exercida conforme a descrição de cada ocupação contida na Classificação Brasileira de Ocupações (CBO ${ }^{21}$.

Nas POFs, o mesmo procedimento de classificação foi adotado, considerando-se, ainda, a atividade econômica que o entrevistado executava, classificada de acordo com a Classificação Nacional de Atividades Econômicas (Cnae) determinada pela Comissão Nacional de Classificações ${ }^{22}$; sendo possível, portanto, estabelecer o Nafo individual em coerência com as classificações realizadas nas pesquisas anteriores.

As informações de ocupação do Endef foram casadas com as categorias da PNSN para 65.937 pessoas, que receberam o Nafo atribuído à ocupação na PNSN. As demais pessoas do Endef tinham ocupações que não ocorreram na PNSN e receberam um Nafo com base na classificação do Endef. Pressupôs-se, para a comparação, que o GE das ocupações não se modificou de forma importante entre as pesquisas e que as características de atividade física de lazer permaneceram constantes para toda a população.

As análises são basicamente descritivas, apresentando a distribuição do Nafo e do EN da população brasileira adulta ocupada à época das pesquisas, segundo sexo, idade e macrorregião. As estimativas de cada pesquisa foram obtidas a partir dos microdados fornecidos pelo IBGE, usando os respectivos pesos amostrais calibrados (para população brasileira por sexo e idade na data de referência de cada pesquisa) em um microcomputador, por meio do SAS ${ }^{\circledR}$.

\section{Resultados}

Os dados apontam um aumento na população brasileira adulta ocupada ao longo do tempo. No Endef, a população estudada correspondeu a 26,4 milhões de adultos (61,1\% da população total). Na PNSN houve aumento da população adulta ocupada, chegando a 49,2 milhões (65,4\% da população), tendência que continuou nas POFs seguintes: 70,2 milhões de ocupados (67,3\% da população) na POF2002 e 96 milhões (68,5\% da população) na POF2008. Com relação à situação do domicílio urbano ou rural, percebe-se, ao longo das pesquisas, aumento no percentual da população adulta ocupada na situação urbana (Endef: 61,9\%; PNSN: 65,7\%; POF-2002: 83,6\%; POF-2008: 82,8\%).

A Figura 1 apresenta a porcentagem de adultos com ocupação declarada por sexo nas pesquisas nacionais. Verifica-se que aproximadamente $80 \%$ da população masculina estava ocupada, sendo isso uma constante desde a década de 1970. Entretanto, o percentual de mulheres com ocupação declarada é crescente, apresentando curva ascendente e variação de mais de 17 pontos percentuais de 1974 a 2009 .

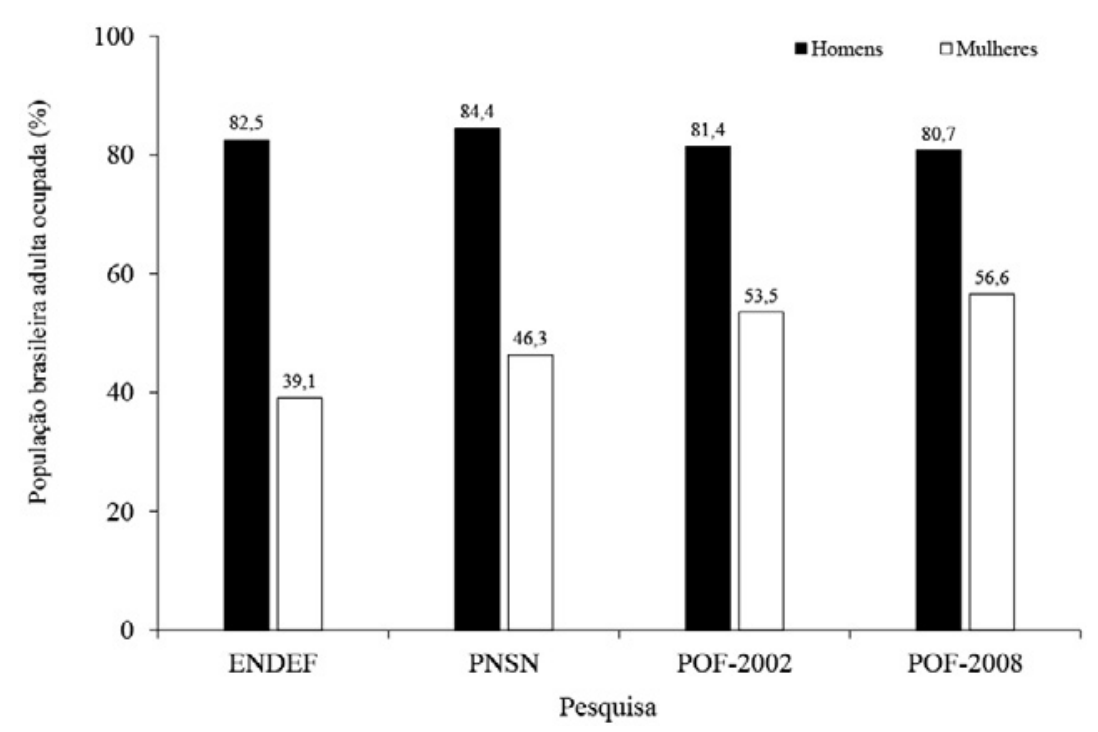

Figura 1 Distribuição (\%) da população brasileira adulta com ocupação declarada, segundo sexo, em pesquisas nacionais das décadas de 1970 a 2000

Endef: Estudo Nacional da Despesa Familiar (dados coletados entre 1974 e 1975).

PNSN: Pesquisa Nacional de Saúde e Nutrição (dados coletados em 1989).

POF-2002: Pesquisa de Orçamentos Familiares (dados coletados entre 2002-2003).

POF-2008: Pesquisa de Orçamentos Familiares (dados coletados entre 2008-2009). 
A distribuição de Nafo em cada uma das pesquisas, segundo sexo e situação urbana ou rural, é apresentada na Tabela 1. A maior frequência de Nafo variou de moderado na década de 1970 e 1980 ( $48,2 \%$ no Endef e $48,6 \%$ na PNSN) para leve nos anos 2000 (44,3\% na POF-2002 e 46,8\% na POF2008). Mais homens desenvolviam atividade de Nafo moderado (45,6\% no Endef e 48,7\% na PNSN), sendo a intensidade do trabalho posteriormente distribuída entre atividades de Nafo leve e moderado (39,0 e $33,7 \%$, respectivamente, na POF-2008). As maiores modificações entre o Endef e a POF-2008 ocorreram nas mulheres, com o aumento do Nafo leve (de 35,9 para $57,6 \%$ ) e pesado (de 10,2 para $24,6 \%$ ) e redução no Nafo moderado (de 53,9 para $17,8 \%$ ).
Sobre a situação do domicílio, há uma grande diferença entre as pesquisas, no que tange ao trabalho em áreas urbanas e rurais (Tabela 1). O trabalho que, até o final dos anos 1980, era predominantemente moderado, 46,0 e 51,8\% em áreas urbanas e rurais, respectivamente, apresentou grandes mudanças. Atualmente, o trabalho urbano é caracterizado por alta frequência de Nafo leve (52,9\% na POF-2008), enquanto na situação rural aumentou a frequência de trabalhadores com Nafo pesado (67,0\% na POF-2008). O aumento na frequência de Nafo leve é progressivo tanto em mulheres quanto em homens em ambas as áreas urbanas e rurais. Já a frequência de Nafo pesado aumentou em todas as combinações exceto para os homens de regiões urbanas em quem houve redução da frequência.

Tabela 1 Nível de atividade física ocupacional (Nafo) da população adulta ocupada no Brasil, segundo sexo e situação de domicílio, em pesquisas nacionais das décadas de 1970 a 2000

\begin{tabular}{|c|c|c|c|c|c|}
\hline & \multirow{2}{*}{ Nafo } & \multicolumn{4}{|c|}{ Pesquisa } \\
\hline & & Endef & PNSN & POF-2002 & POF-2008 \\
\hline \multirow[t]{3}{*}{ Total } & Leve & 26,6 & 28,8 & 44,3 & 46,8 \\
\hline & Moderado & 48,2 & 48,6 & 28,0 & 27,1 \\
\hline & Pesado & 25,2 & 22,6 & 27,7 & 26,1 \\
\hline \multirow[t]{3}{*}{ Homens } & Leve & 22,3 & 21,4 & 37,8 & 39,0 \\
\hline & Moderado & 45,6 & 48,7 & 34,3 & 33,7 \\
\hline & Pesado & 32,1 & 29,9 & 27,9 & 27,3 \\
\hline \multirow[t]{3}{*}{ Mulheres } & Leve & 35,9 & 42,4 & 53,9 & 57,6 \\
\hline & Moderado & 53,9 & 48,4 & 18,6 & 17,8 \\
\hline & Pesado & 10,2 & 9,2 & 27,5 & 24,6 \\
\hline \multirow[t]{3}{*}{ Urbano } & Leve & 39,1 & 35,5 & 50,3 & 52,9 \\
\hline & Moderado & 46,0 & 49,0 & 30,8 & 29,4 \\
\hline & Pesado & 14,9 & 15,5 & 18,9 & 17,7 \\
\hline \multirow[t]{3}{*}{ Rural } & Leve & 6,3 & 6,5 & 13,8 & 17,2 \\
\hline & Moderado & 51,8 & 46,8 & 13,9 & 15,8 \\
\hline & Pesado & 41,9 & 46,7 & 72,3 & 67,0 \\
\hline Urbano & Leve & 34,4 & 27,8 & 43,8 & 45,5 \\
\hline \multirow[t]{2}{*}{ Homens } & Moderado & 44,1 & 48,8 & 38,8 & 38,0 \\
\hline & Pesado & 21,5 & 23,4 & 17,4 & 16,5 \\
\hline Urbano & Leve & 48,1 & 47,6 & 59,4 & 62,6 \\
\hline \multirow[t]{2}{*}{ Mulheres } & Moderado & 49,7 & 49,4 & 19,4 & 18,3 \\
\hline & Pesado & 2,2 & 3,0 & 21,2 & 19,1 \\
\hline Rural & Leve & 4,3 & 3,9 & 10,9 & 12,8 \\
\hline \multirow[t]{2}{*}{ Homens } & Moderado & 47,8 & 48,0 & 14,1 & 16,3 \\
\hline & Pesado & 47,9 & 48,1 & 75,0 & 70,9 \\
\hline Rural & Leve & 11,6 & 14,7 & 19,5 & 26,1 \\
\hline \multirow[t]{2}{*}{ Mulheres } & Moderado & 62,2 & 43,2 & 13,6 & 14,9 \\
\hline & Pesado & 26,2 & 42,1 & 66,9 & 59,0 \\
\hline
\end{tabular}

Endef: Estudo Nacional da Despesa Familiar (dados coletados entre 1974 e 1975).

PNSN: Pesquisa Nacional de Saúde e Nutrição (dados coletados em 1989).

POF-2002: Pesquisa de Orçamentos Familiares (dados coletados entre 2002-2003).

POF-2008: Pesquisa de Orçamentos Familiares (dados coletados entre 2008-2009). 
Ao se analisar a distribuição do Nafo por macrorregião (dados não mostrados em tabela), a região Norte do país apresentou tendência à estabilização do Nafo leve (40,3\% na POF-2008), com o Nafo pesado quase dobrando (de $16,8 \%$ na PNSN para $32,8 \%$ na POF-2008) e o Nafo moderado caindo pela metade (de $50,7 \%$ na PNSN para $26,6 \%$ na POF2008). Na região Centro-Oeste houve aumento do Nafo leve (de 38,1\% no Endef para 52,5\% na POF2008), redução do moderado (de $46,4 \%$ no Endef para $26,4 \%$ na POF-2008) e discreto aumento com tendência à estabilização do Nafo pesado (de 15,6\% no Endef para 21,1\% na POF-2008). Já na região Sul, o Nafo leve mais que dobrou (de 21,4\% no Endef para $47,6 \%$ na POF-2008), tendo diminuição no moderado (de 48,1\% no Endef para 27,6\% na POF2008 ) e pequena variação no pesado (de $30,5 \%$ no Endef para 24,8\% na POF-2008). Nas regiões Nordeste e Sudeste houve aumento substancial no Nafo leve e diminuição do moderado, já o Nafo pesado aumentou no Nordeste e se estabilizou no Sudeste. Vale lembrar que tanto no Endef quanto na PNSN a área rural da região Norte não foi pesquisada. Da mesma forma não se pesquisou a área rural da região Centro-Oeste no Endef.

A Figura 2 apresenta a distribuição do EN do conjunto da população brasileira por sexo e para o Nafo leve e pesado em cada uma das pesquisas. Pode-se observar que ao longo do intervalo entre as pesquisas, houve redução de aproximadamente $3 / 4$ no baixo peso para a população total (de $9,8 \%$ no Endef para 2,3\% na POF-2008) e pelo menos duplicação na prevalência de sobrepeso na população como um todo (de 22,6\% no Endef para 48,2\% na POF-2008) e em homens (de 17,9\% no Endef para $50,4 \%$ na POF-2008). Nas mulheres houve aumento (de $27,4 \%$ no Endef para $45,2 \%$ na POF-2008), mas não tão acentuado quando comparado aos homens. Na população masculina (Figura 3), nota-se queda acentuada do peso adequado no Nafo leve de 64,1 para $41,6 \%$ e aumento no sobrepeso de 30,5 para $57,1 \%$. Para o Nafo pesado observa-se situação semelhante, o peso adequado variando de 81,1 para $58,6 \%$. O sobrepeso variou de 11,5 para $39,4 \%$ no mesmo período. Percebe-se que na POF-2002 os percentuais de peso adequado e sobrepeso eram praticamente iguais (49,7 e 48,3\%), com inversão a partir deste momento, quando o sobrepeso tornou-se mais prevalente entre os homens trabalhadores com atividade de Nafo leve.

No caso da população feminina (Figura 3), percebe-se que, entre o Endef e a POF-2008 e em atividades de Nafo leve, houve queda no peso adequado de 65,6 para $54,9 \%$ e aumento no sobrepeso de 23,7 para $41,9 \%$. No Nafo pesado, o peso adequado caiu de 64,0 para $47,0 \%$ e o sobrepeso aumentou de 20,6 para $49,5 \%$, sendo esta a curva mais acentuada observada em mulheres. Os dados da POF-2008 apontam que o sobrepeso $(49,5 \%)$ se tornou mais prevalente do que o peso adequado $(47,0 \%)$ em atividades de Nafo pesado. Ainda com relação ao sobrepeso, no Nafo pesado é importante ressalvar que, nas duas primeiras pesquisas, ele era menor que o sobrepeso no Nafo leve, porém após a PNSN ambos tiveram crescimento, tendo o primeiro uma curva mais ascendente, com variação de $10 \%$ entre 2002 e 2009.

Quando observados o Nafo e o EN nas macrorregiões do país ao longo do período analisado, os achados corroboram as tendências apresentadas para o conjunto da população brasileira.

A prevalência de sobrepeso da população brasileira adulta ocupada teve variação de 27,6 para $49,3 \%$ na categoria de Nafo leve e de 12,7 para $43,3 \%$ no Nafo pesado, ambas do Endef para a POF2008. Por outro lado, houve redução no baixo peso em ambas as categorias de Nafo (de 7,7 para 2,3\% e de 8,5 para $2,6 \%$ entre as categorias de Nafo leve e pesado do Endef para a POF-2008, respectivamente). Essa tendência de mudança no perfil nutricional foi observada tanto em homens quanto em mulheres independentemente do Nafo, mas com características diferentes entre os sexos (Tabela 2).

O baixo peso, que era situação crítica na década de 1970, teve alterações importantes. Nas regiões Norte e Centro-Oeste, o baixo peso para Nafo leve, 9,5 e 9\%, respectivamente, que ultrapassava o limite crítico de 5\% compatível com indivíduos constitucionalmente magros ${ }^{6}$, caiu para 2,2 e $2,5 \%$ na POF2008. No Nordeste, o baixo peso nos Nafos leve e pesado diminuiu 7\% no período. Nas regiões Norte e Sul havia maior prevalência de peso adequado para o Nafo leve na década de 1970. No final dos anos 2000, a prevalência de sobrepeso em atividades leves suplantou o baixo peso e o peso adequado, atingindo 50,6 e 51,9\%, respectivamente. No caso da região Sul, isso também foi observado no Nafo pesado, com a prevalência quase triplicando $(18,9$ para 52,3\% do Endef para a POF-2008). Interessante notar que o aumento do sobrepeso entre as pesquisas ficou em torno de duas vezes para o país como um todo, mas variou bastante entre as macrorregiões, sendo de 2,7 vezes na região Norte e 4,5 vezes no Nordeste para a categoria de Nafo pesado (Tabela 2). 


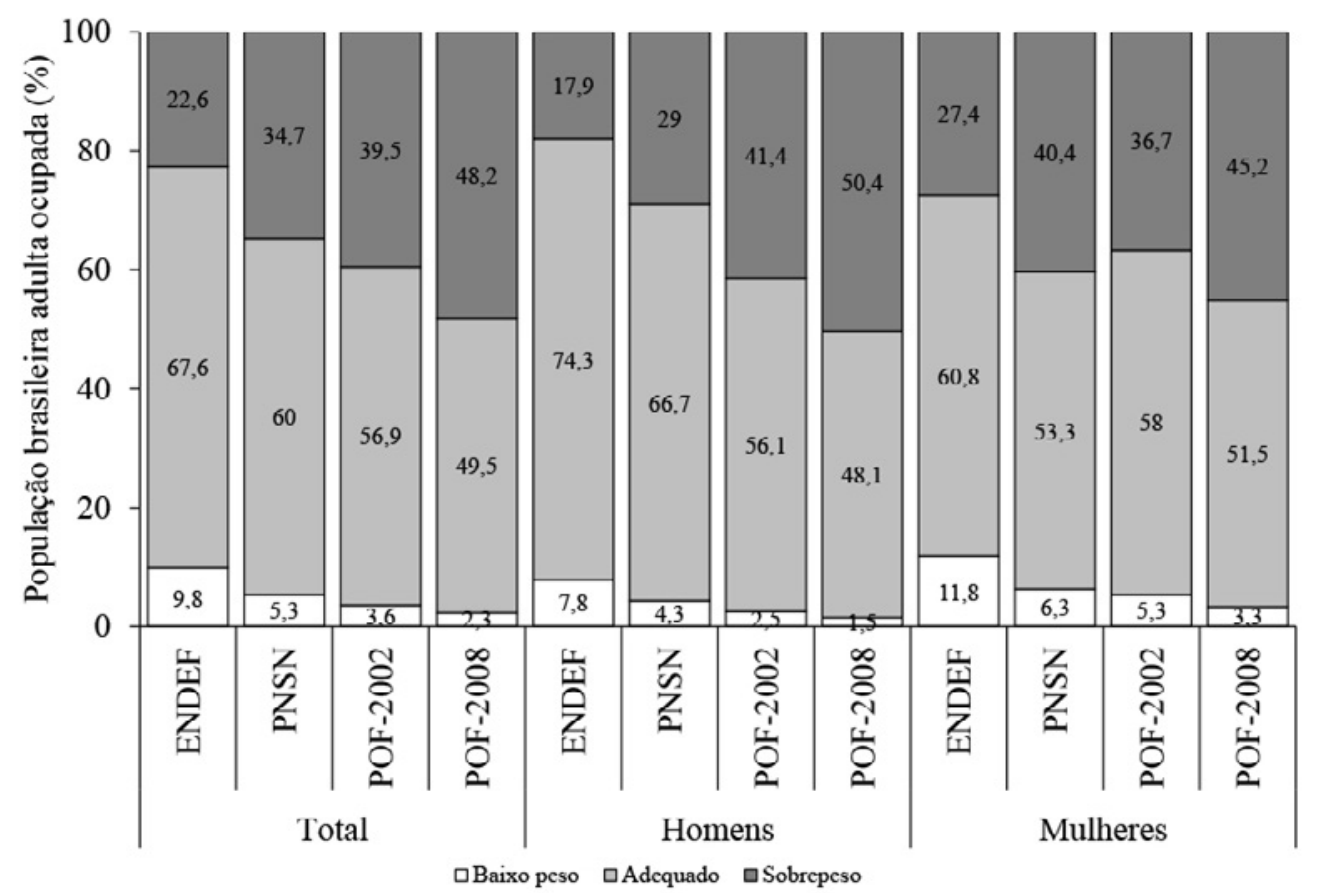

Figura 2 Estado nutricional da população brasileira adulta ocupada, segundo pesquisas nacionais das décadas de 1970 a 2000 Endef: Estudo Nacional da Despesa Familiar (dados coletados entre 1974 e 1975). PNSN: Pesquisa Nacional de Saúde e Nutrição (dados coletados em 1989).

POF-2002: Pesquisa de Orçamentos Familiares (dados coletados entre 2002-2003). POF-2008: Pesquisa de Orçamentos Familiares (dados coletados entre 2008-2009).

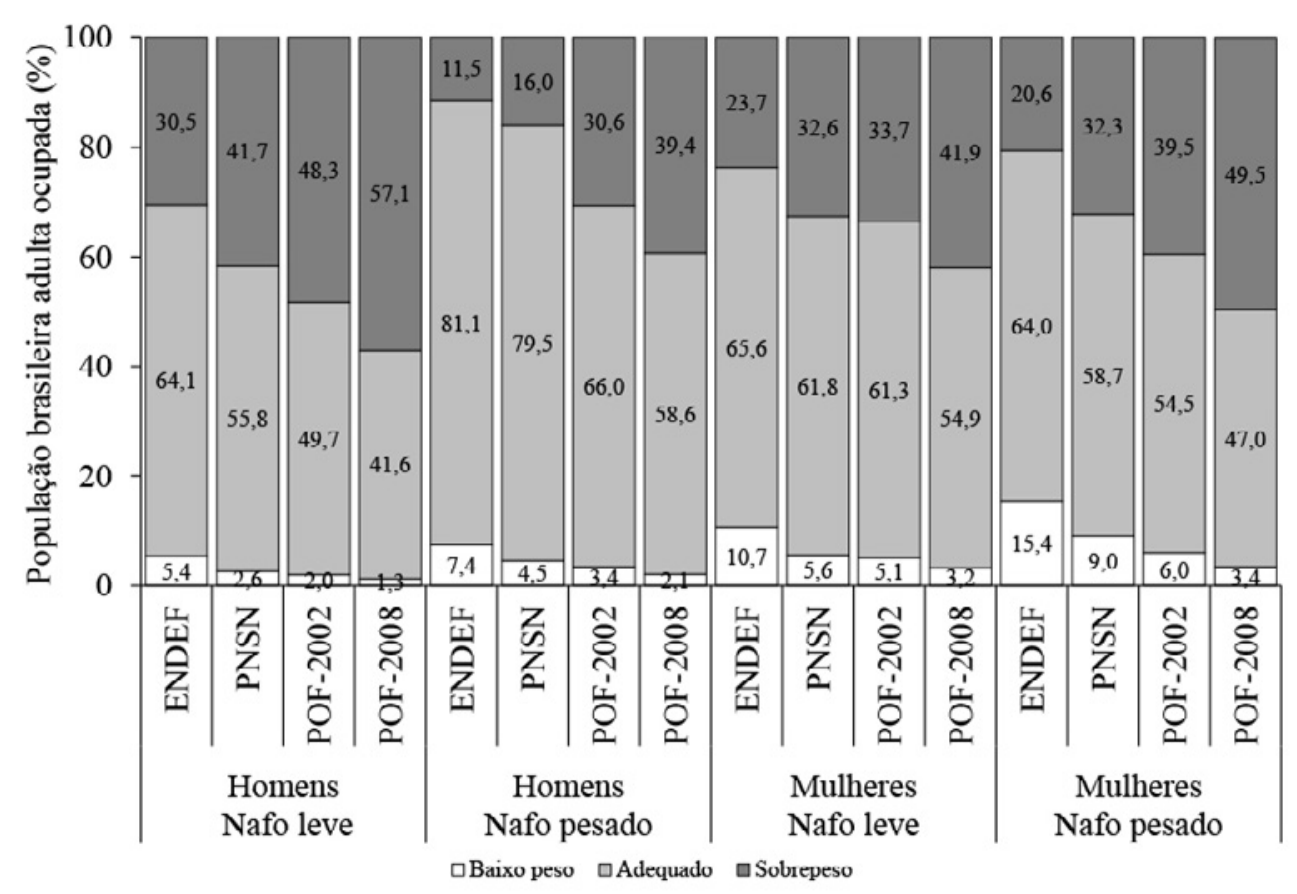

Figura 3 Estado nutricional da população adulta ocupada brasileira masculina e feminina, segundo nível de atividade física ocupacional ( $\mathrm{Nafo}$ ) leve e pesado, em pesquisas nacionais das décadas de 1970 a 2000

Endef: Estudo Nacional da Despesa Familiar (dados coletados entre 1974 e 1975).

PNSN: Pesquisa Nacional de Saúde e Nutrição (dados coletados em 1989).

POF-2002: Pesquisa de Orçamentos Familiares (dados coletados entre 2002-2003).

POF-2008: Pesquisa de Orçamentos Familiares (dados coletados entre 2008-2009). 
Tabela 2 População brasileira adulta com ocupação declarada, segundo macrorregião, estado nutricional e nível de atividade física ocupacional (Nafo) leve e pesado, nas pesquisas nacionais das décadas de 1970 a 2000

\begin{tabular}{|c|c|c|c|c|c|c|c|c|}
\hline \multirow{3}{*}{$\begin{array}{c}\text { Macrorregiãoe } \\
\text { estado nutricional }\end{array}$} & \multicolumn{8}{|c|}{ Nafo } \\
\hline & \multicolumn{4}{|c|}{ Leve } & \multicolumn{4}{|c|}{ Pesado } \\
\hline & Endef & PNSN & POF-2002 & POF-2008 & Endef & PNSN & POF-2002 & POF-2008 \\
\hline \multicolumn{9}{|l|}{ Brasil } \\
\hline Baixo peso & 7,7 & 4,1 & 3,5 & 2,3 & 8,5 & 5,1 & 4,4 & 2,6 \\
\hline Adequado & 64,7 & 58,9 & 55,3 & 48,4 & 78,8 & 76,5 & 61,4 & 54,1 \\
\hline Sobrepeso & 27,6 & 37,0 & 41,2 & 49,3 & 12,7 & 18,4 & 34,2 & 43,3 \\
\hline \multicolumn{9}{|l|}{ Norte } \\
\hline Baixo peso & 9,5 & 3,8 & 2,9 & 2,2 & 4,0 & 4,2 & 4,0 & 2,7 \\
\hline Adequado & 64,0 & 57,7 & 57,6 & 47,2 & 79,8 & 75,1 & 66,0 & 54,0 \\
\hline Sobrepeso & 26,5 & 38,5 & 39,5 & 50,6 & 16,2 & 20,7 & 30,0 & 43,3 \\
\hline \multicolumn{9}{|l|}{ Nordeste } \\
\hline Baixo peso & 10,4 & 4,8 & 4,6 & 3,6 & 10,1 & 5,8 & 5,1 & 3,3 \\
\hline Adequado & 65,9 & 62,1 & 56,2 & 49,3 & 81,6 & 82,1 & 67,2 & 59,5 \\
\hline Sobrepeso & 23,7 & 33,1 & 39,2 & 47,1 & 8,3 & 12,1 & 27,7 & 37,2 \\
\hline \multicolumn{9}{|l|}{ Sudeste } \\
\hline Baixo peso & 7,7 & 4,5 & 3,4 & 1,9 & 9,6 & 5,9 & 4,4 & 2,3 \\
\hline Adequado & 64,9 & 58,3 & 54,5 & 48,6 & 77,5 & 74,7 & 57,7 & 51,8 \\
\hline Sobrepeso & 27,4 & 37,2 & 42,1 & 49,5 & 12,9 & 19,4 & 37,9 & 45,9 \\
\hline \multicolumn{9}{|l|}{ Sul } \\
\hline Baixo peso & 3,7 & 2,5 & 2,4 & 1,6 & 4,6 & 2,5 & 3,2 & 1,5 \\
\hline Adequado & 62,2 & 57,0 & 55,5 & 46,5 & 76,5 & 69,4 & 55,5 & 46,2 \\
\hline Sobrepeso & 34,1 & 40,5 & 42,1 & 51,9 & 18,9 & 28,1 & 41,3 & 52,3 \\
\hline \multicolumn{9}{|l|}{ Centro-Oeste } \\
\hline Baixo peso & 9,0 & 3,9 & 4,0 & 2,5 & 6,4 & 3,7 & 4,8 & 2,5 \\
\hline Adequado & 66,7 & 60,2 & 56,4 & 49,6 & 82,1 & 79,3 & 59,8 & 49,2 \\
\hline Sobrepeso & 24,3 & 35,9 & 39,6 & 47,9 & 11,5 & 17,0 & 35,4 & 48,3 \\
\hline
\end{tabular}

Endef: Estudo Nacional da Despesa Familiar (dados coletados entre 1974 e 1975).

PNSN: Pesquisa Nacional de Saúde e Nutrição (dados coletados em 1989).

POF-2002: Pesquisa de Orçamentos Familiares (dados coletados entre 2002-2003).

POF-2008: Pesquisa de Orçamentos Familiares (dados coletados entre 2008-2009).

$\mathrm{IMC}=$ índice de massa corporal; baixo peso $\left(\mathrm{IMC}<18,5 \mathrm{~kg} \cdot \mathrm{m}^{-2}\right)$; adequado $\left(18,5 \mathrm{~kg} \cdot \mathrm{m}^{-2} \leq \mathrm{IMC}<25 \mathrm{~kg} \cdot \mathrm{m}^{-2}\right)$ e sobrepeso $\left(\mathrm{IMC} \geq 25 \mathrm{~kg} \cdot \mathrm{m}^{-2}\right)$.

\section{Discussão}

A característica demográfica brasileira é bem evidenciada na comparação entre as quatro pesquisas, decorrente do aumento da população urbana no país, comprovado pelos dados dos censos demográficos brasileiros: em 1970, 52,9 milhões de habitantes residindo em áreas urbanas (55,9\% da população total); em 1991, 110,9 milhões de brasileiros em áreas urbanas (75,5\% da população total); e em 2010, 160,9 milhões habitantes concentravam-se em áreas urbanas, representando $84,3 \%$ da população do país ${ }^{23,24}$. Esse aumento expressivo da população urbana é consequência de três fatores: o próprio crescimento vegetativo nas áreas urbanas; a migração de áreas rurais para urbanas e a incorporação de áreas que antes eram consideradas rurais em função das alterações legais dos perímetros urbanos das cidades e vilas, visto que os conceitos de urbano e rural do IBGE seguem a delimitação dos perímetros urbanos estabelecida na legislação municipal. 
Apesar do crescimento da população adulta ocupada em todo o país, percebe-se aumento substancial na proporção da população urbana ocupada e redução importante na população rural ocupada entre as pesquisas. Esse perfil influencia tanto o estilo de vida (alimentação e atividades de lazer) quanto o custo energético do trabalho já que se pode imaginar que as ocupações das áreas urbanas, predominantemente industriais e de serviços, demandem menos energia para seu exercício do que as das áreas rurais, voltadas mais para o setor agropecuário ${ }^{25}$.

A população brasileira, à semelhança de populações de outros países em desenvolvimento, vive um período de transição nutricional ${ }^{26}$, que no caso brasileiro é bastante intenso, com expressivo aumento nas prevalências de sobrepeso e redução nas taxas de baixo peso, como evidenciado nas comparações entre as quatro pesquisas ${ }^{6}$. A novidade nesta análise é o fato dessa tendência ocorrer tanto na categoria de Nafo leve quanto na pesada, entre homens e mulheres em todas as macrorregiões do país. Em comparação com o Endef, a prevalência de sobrepeso na POF-2008 aumentou em ritmo maior na categoria Nafo pesado do que na leve. Esse fato parece indicar crescente balanço energético positivo em toda a população pesquisada que poderia ser decorrente de aumento na IE ou redução no GE geral, ou a combinação de ambos os fatores. Não existem dados sobre a tendência da ingestão energética na população em geral, entretanto dados de disponibilidade de energia para consumo da população brasileira oriundos da Folha de Balanço Alimentar ${ }^{27}$ indicam aumento de aproximadamente $188 \mathrm{kcal}$ por dia per capita por década entre o Endef e a POF-2002 ${ }^{28}$.

Nas POFs, foram coletados dados de consumo alimentar e das características da população ${ }^{29}$. O consumo, na maioria das pesquisas, foi levantado a partir da aquisição de alimentos, ou seja, quanto de alimento foi adquirido para o domicílio, não sendo computados os estoques alimentares - que permitiriam estimar a disponibilidade de alimentos para consumo no domicílio - nem os alimentos ingeridos fora do domicílio, o que pode configurar uma subestimativa da ingestão alimentar ${ }^{28}$. Apenas na POF mais recente investigou-se a ingestão alimentar a partir de registro alimentar ${ }^{30}$. Se, por um lado, esta pesquisa foi um avanço no que tange à avaliação da IE da população, por outro, ainda não há no Brasil pesquisa nacional sobre estimativa do NAF e do GE da população. Isso é fundamental, já que o sedentarismo, ou a inatividade física, fenômeno internacionalmente considerado como uma epidemia, identificado como o quarto fator de risco para a mortalidade global ${ }^{31}$ e definido como a não realização de atividade física dentro do recomendado, é, muitas vezes, apontado como o componente mais importante na explicação do aumento de sobrepeso/obesidade em sociedades do primeiro mundo ${ }^{32}$, visto que se supõe que a AFO está reduzindo nas últimas décadas e seja menor nestas sociedades ${ }^{2}$.

Mais recentemente, estabeleceu-se, no país, o sistema de Vigilância de Fatores de Risco e Proteção para Doenças Crônicas por Inquérito Telefônico (Vigitel), sob o patrocínio do Ministério da Saúde, ocorrido anualmente desde 2006 e no qual são avaliados o EN e alguns marcadores de atividade física e da dieta da população com idade superior a 10 anos. $\mathrm{O}$ dado mais recente ${ }^{33}$, referente ao conjunto das 27 capitais e do Distrito Federal em 2014, indica aumento de $23 \%$ na prevalência de sobrepeso nos últimos nove anos, totalizando $52,5 \%$ da população com idade superior a 18 anos e maior em homens $(56,5 \%)$ do que em mulheres $(49,1 \%)$. Já a obesidade está presente em $17,9 \%$ da população, sendo levemente superior em mulheres. Os dados de atividade física do Vigitel de $2014^{33}$ indicam que cerca de $35 \%$ do conjunto da população se considerou ativa por realizar o equivalente a 150 minutos de atividade moderada no tempo livre representando aumento de $18 \%$ nos seis anos anteriores. No total, $15 \%$ eram fisicamente inativos e $49 \%$ insuficiente ativos nos quatro domínios da atividade física: doméstico, ocupacional, lazer ou transporte. Tanto as informações antropométricas quanto as sobre a atividade física são autorrelatadas, o que pode introduzir vieses nas estimativas se comparadas com as medições diretas. As atividades ocupacionais avaliam a frequência e o tempo realizando atividades pesadas ou se o entrevistado anda bastante a pé no trabalho.

Mesmo nas populações de países desenvolvidos, a falta de uso das informações ocupacionais (e domésticas) deve-se à indisponibilidade de tais informações, principalmente pelo fato das pessoas terem maior controle de como elas gastam seu tempo no lazer. Assim, a medição somente da atividade física de lazer pode levar a uma subestimação da atividade física total, especialmente nos indivíduos com ocupações intensas, mesmo em países desenvolvidos ${ }^{34,35}$. De fato, o emprego de medidas objetivas da atividade física (AF) permite estabelecer a importância da AFO na AF total dos indivíduos, também em países desenvolvidos. Por exemplo, a comparação entre os indivíduos empregados com os desempregados em inquérito nacional nos Estados Unidos indicou que os empregados ficavam mais tempo em atividades de intensidade moderada e pesada do que os não empregados. Entretanto, em inquérito semelhante realizado na Suécia ${ }^{36}$ o mesmo resultado não foi encontrado. Porém, em ambos os países, a AFO esteve associada com a AF total diária entre os indivíduos empregados. Indivíduos que realizam menos $\mathrm{AF}$ durante o trabalho parecem realizar menos AF também fora do trabalho ${ }^{37}$. A premissa de que trabalhadores que realizam AFO pesada possam, de forma isolada, ter menor risco de adoecer deve ser 
olhada de forma cuidadosa. Recente estudo realizado em amostra populacional americana (NHANES III) evidenciou que a AFO estava associada positivamente com todas as causas de morte ${ }^{38}$.

Com a ressalva de diferença de métodos na classificação das ocupações pelo CBO/Comissão Nacional de Classificações ocorrida em 2002, houve maior inserção das mulheres no mercado de trabalho no Brasil, fenômeno que merece destaque. Alguns autores têm ressaltado que, na realidade, o que está sendo observado é uma maior visibilidade do trabalho feminino, antes restrito a afazeres domésticos e trabalhos familiares não remunerados ${ }^{39,40}$. Por exemplo, na década de 1990, no estado de São Paulo houve expansão do nível ocupacional entre as mulheres residentes rurais, com a criação de 36 mil ocupações no período ${ }^{41}$.

Pesquisas realizadas pelo IBGE a partir dos anos 2000, com novos métodos de investigação como a Pesquisa Mensal de Empregos ${ }^{42}$, mostram um maior número de trabalhadores domésticos legalizados, trabalho predominantemente feminino $(94,5 \% \mathrm{em}$ 2009) e de aparente Nafo pesado. O trabalho realizado dentro do domicílio possibilita o acesso a alimentos com maior frequência, sendo talvez essa uma das razões para o sobrepeso mesmo quando da prática de trabalho de nível pesado.

Os achados deste estudo assemelham-se aos resultados de pesquisa longitudinal sobre a AFO e a obesidade na população americana ${ }^{2}$. Os autores estimaram que o GE diário relacionado com a ocupação diminuiu mais de $100 \mathrm{kcals}$, sendo essa redução parte do motivo para o aumento da massa corporal médio em ambos os sexos.

\section{Conclusão}

Esta análise permite concluir que houve aumento na prevalência de sobrepeso e redução na prevalência de baixo peso na subpopulação adulta com ocupação declarada entre 1974 e 2009. Esse fenômeno foi encontrado, em ritmos diferentes, em todas as macrorregiões do país, em todas as categorias de AFO e tanto em mulheres quanto em homens. Assim, pode-se concluir, ainda, que o Nafo parece não explicar o acentuado aumento na prevalência de sobrepeso na subpopulação brasileira estudada. Há pelo menos três possíveis explicações para este fato: (1) aumento da ingestão energética por adulto no período; (2) redução do custo energético das atividades ocupacionais consideradas, talvez devido a modificações nos processos de trabalho; (3) modificação no nível de atividade física de lazer (não laboral). É fundamental que as próximas pesquisas nacionais possam coletar dados mais detalhados sobre as características do trabalho e do lazer, de preferência utilizando medidas objetivas de AF, para uma melhor compreensão da relação entre o EN e a AF na população brasileira adulta ocupada.

\section{Contribuições de autoria}

Todos os autores participaram da concepção do estudo, da análise e interpretação dos dados, da redação e da aprovação da versão final do manuscrito publicado.

\section{Referências}

1. Popkin BM, Adair LS, Ng SW. Global nutrition transition and the pandemic of obesity in developing countries. Nutr Rev. 2012;70(1):3-21.

2. Church TS, Thomas DM, Tudor-Locke C, Katzmarzyk PT, Earnest, CP, Rodarte RQ, et al. Trends over 5 decades in U.S. occupation-related physical activity and their associations with obesity. PLoS ONE. 2011;6(5):e19657.

3. World Health Organization. 10 facts on obesity [Internet]. 2018 [acesso em 2018 may 8]. Disponível em: http://www.who.int/features/ factfiles/obesity/en/

4. Mendonça PC, Anjos LA. Aspectos das práticas alimentares e da atividade física como determinantes do crescimento do sobrepeso/ obesidade no Brasil. Cad Saúde Pública. 2004;20(3):698-709.
5. Instituto Brasileiro de Geografia e Estatística. Pesquisa de Orçamentos Familiares 2002-2003. Antropometria e estado nutricional de crianças, adolescentes e adultos no Brasil. Rio de Janeiro: IBGE; 2004.

6. Instituto Brasileiro de Geografia e Estatística. Pesquisa de Orçamentos Familiares 2008-2009. Antropometria e estado nutricional de crianças, adolescentes e adultos no Brasil. Rio de Janeiro: IBGE; 2010.

7. Shook RP, Hand GA, Blair SN. Top 10 research questions related to energy balance. Res Q Exerc Sport. 2014;85(1):49-58.

8. Caspersen CJ, Powell KE, Christensen GM. Physical activity, exercise, and physical fitness: definitions and distinctions for health-related research. Public Health Rep. 1985;100(2):126-31. 
9. Monda KL, Adair LS, Zhai F, Popkin BM. Longitudinal relationships between occupational and domestic physical activity patterns and body weight in China. Eur J Clin Nutr. 2008;62(11):1318-25.

10. Pan American Health Organization. Ultraprocessed food and drink products in Latin America: Trends, impact on obesity, policy implications [Internet]. Washington, DC: PAHO; 2015 [2018 abr 30]. Disponível em: http://www. paho.org/hq/index.php?option =com_content\&view $=$ article\&id $=11153 \&$ Itemid $=0 \& l a n g=e s$

11. Monteiro CA, Mondini L, Costa R. Mudanças na composição e adequação nutricional da dieta familiar nas áreas metropolitanas do Brasil (19881996). Rev Saúde Pública. 2000;34(3):251-8.

12. Hallal PC, Anjos LA. Epidemiologia da atividade física. In: Kac G, Sichieri R, Gigante DP, organizadores. Epidemiologia nutricional. Rio de Janeiro: Fiocruz; Atheneu; 2007. p. 461-72.

13. Vasconcellos MTL. Metodologia do Estudo Nacional da Despesa Familiar: objetivos descrição e metodologia usada no Endef. Rio de Janeiro: IBGE; Endef; 1983. V. 3.

14. Instituto Nacional de Alimentação e Nutrição. Pesquisa Nacional sobre Saúde e Nutrição. Resultados Preliminares. 2. ed. Brasília, DF: INAN; 1990.

15. Instituto Brasileiro de Geografia e Estatística. Pesquisa de Orçamentos Familiares 2002-2003. Primeiros resultados. Brasil e grandes regiões. 2. ed. Rio de Janeiro: IBGE; 2004.

16. Instituto Brasileiro de Geografia e Estatística. Pesquisa de Orçamentos Familiares 2008-2009. Despesas, rendimentos e condições de vida. Rio de Janeiro: IBGE; 2010.

17. World Health Organization. Obesity: preventing and managing the global epidemic. Geneva: WHO; 2000. WHO Technical Report Series, n. 894.

18. Vasconcellos MTL. Análise crítica dos métodos de avaliação nutricional de populações, a partir de dados de consumo familiar de energia [tese]. Rio de Janeiro: Escola Nacional de Saúde Pública; Fundação Oswaldo Cruz; 2001.

19. Food and Agriculture Organization of the United Nations. World Health Organization. Energy and protein requirements. Report of a Joint FAO/WHO ad hoc Expert Consultation. Rome: FAO; 1973. FAO Nutrition Meeting Report Series, n. 52.

20. Food and Agriculture Organization of the United Nations. World Health Organization. United Nations University. Energy and protein requirements. Report of a Joint FAO/WHO/UNU Expert Consultation. Geneva: WHO; 1985. WHO Technical Report Series, n. 724.

21. Ministério do Trabalho. Classificação Brasileira de Ocupações. Sistema Nacional de Emprego. Brasília, DF: Ministério do Trabalho; 1982.
22. Instituto Brasileiro de Geografia e Estatística. CNAE 1.0. Classificação Nacional de Atividades Econômicas. Rio de Janeiro: Concla; IBGE; 2002.

23. Instituto Brasileiro de Geografia e Estatística. Censo demográfico 1991. Resultados preliminares. Rio de Janeiro: IBGE; 1992.

24. Instituto Brasileiro de Geografia e Estatística. Censo demográfico de 2010. Resultados gerais da amostra. Rio de Janeiro: IBGE; 2012.

25. James WPT, Schofield EC. Human energy requirements: a manual for planners and nutritionists. New York: FAO; Oxford University Press; 1990.

26. Popkin BM. Nutrition transition and the global diabetes epidemic. Curr Diab Rep. 2015;15(9):64.

27. Anjos LA, Wahrlich V. Recomendações de energia e prevenção de doenças. In: Dolinsky M, editor. Recomendações nutricionais e prevenção de doenças. São Paulo: Roca; 2011. p. 1-18.

28. Anjos LA, Souza DR, Rossato SL. Desafios na medição quantitativa da ingestão alimentar em estudos populacionais. Rev Nutr. 2009;22(1): 151-61.

29. Instituto Brasileiro de Geografia e Estatística. Pesquisa de Orçamentos Familiares 2008-2009. Aquisição alimentar domiciliar per capita: Brasil e grandes regiões. Rio de Janeiro: IBGE; 2010.

30. Instituto Brasileiro de Geografia e Estatística. Pesquisa de Orçamentos Familiares 2008-2009. Análise do Consumo Alimentar Pessoal no Brasil. Rio de Janeiro: IBGE; 2010.

31. World Health Organization. Physical inactivity: a global public health problem [Internet]. [acesso em 2015 set 19]. Disponível em: http://www.who.int/ dietphysicalactivity/factsheet_inactivity/en/

32. World Health Organization. Diet, nutrition and prevention of chronic disease. Geneva: WHO; 2003. WHO Technical Report Series, n. 916.

33. Brasil. Vigitel Brasil 2014: vigilância de fatores de risco e proteção para doenças crônicas por inquérito telefônico. Brasília, DF: Ministério da Saúde; Secretaria de Vigilância em Saúde; Departamento de Vigilância de Doenças e Agravos não Transmissíveis e Promoção da Saúde; 2015.

34. U.S. Department of Health and Human Services. Physical activity and health. A report of the surgeon general. Atlanta, GA: DHHS; Centers for Disease Control and Prevention; National Center for Chronic Disease Prevention and Health Promotion; 1996.

35. Centers for Disease Control and Prevention. Contribution of occupational physical activity toward meeting recommended physical activity guidelines: United States, 2007. MMWR Morb Mortal Wkly Rep. 2011;60(20):656-60.

36. Kwak L, Berrigan D, Van Domelen D, Sjöström M, Hagströmer M. Examining differences in physical activity levels by employment status and/ or job activity level: Gender-specific comparisons 
between the United States and Sweden. J Sci Med Sport. 2015 Jun 4. doi: 10.1016/j. jsams.2015.05.008. [Epub ahead of print].

37. JaKa MM, Haapala JL, Wolfson J, French SA. Describing the relationship between occupational and non-occupational physical activity using objective measurement. Prev Med Rep. 2015;2:213-7.

38. Richard A, Martin B, Wanner M, Eichholzer M, Rohrmann S. Effects of leisure-time and occupational physical activity on total mortality risk in NHANES III according to sex, ethnicity, central obesity, and age. J Phys Act Health. 2015;12(2):184-92.

39. Lavinas L. Emprego feminino: o que há de novo e o que se repete [Internet]. Dados. 1997;40(1) [acesso em 2018 maio 2]. Disponível em: https://dx.doi. org/10.1590/S0011-52581997000100003
40. Melo HP. A invisibilidade do trabalho feminino nas estatísticas [Internet]. [acesso em 2015 ago 2]. Disponível em: http://www.ibge.gov.br/ confest_e_confege/pesquisa_trabalhos/CD/mesas redondas/232-1.doc

41. Fundação Sistema Estadual de Análise de Dados. Ocupação: atividades não-agrícolas determinam o crescimento da ocupação entre as residentes rurais. Mulher Trabalho. 2001;6:9-10. Disponível em: https://www.seade.gov.br/produtos/midia/mulhertrabalho/boletim_MuTrab6.pdf

42. Instituto Brasileiro de Geografia e Estatística. Pesquisa mensal de empregos. Mulher no mercado de trabalho: perguntas e respostas [Internet]. Rio de Janeiro: IBGE; 2010 [acesso em 2018 maio 2]. Disponível em: http://www.ibge.gov.br/home/ estatistica/indicadores/trabalhoerendimento/pme nova/Mulher_Mercado_Trabalho_Perg_Resp.pdf. Acessado em 2 de agosto de 2015 